ANNALES

POLONICI MATHEMATICI

LXXVI.3 (2001)

\title{
A monotone method for constructing extremal solutions to second order periodic boundary value problems
}

\author{
by DAqING JiAng (Changchun) and LingBin Kong (Anda)
}

\begin{abstract}
We describe a constructive method which yields two monotone sequences that converge uniformly to extremal solutions to the periodic boundary value problem $u^{\prime \prime}(t)=f\left(t, u(t), u^{\prime}(t)\right), u(0)=u(2 \pi), u^{\prime}(0)=u^{\prime}(2 \pi)$ in the presence of a lower solution $\alpha(t)$ and an upper solution $\beta(t)$ with $\beta(t) \leq \alpha(t)$.
\end{abstract}

1. Introduction and main result. This paper is concerned with the following second order periodic boundary value problem:

$$
\left\{\begin{array}{l}
u^{\prime \prime}(t)=f\left(t, u(t), u^{\prime}(t)\right), \quad t \in[0,2 \pi], \\
u(0)=u(2 \pi), \quad u^{\prime}(0)=u^{\prime}(2 \pi),
\end{array}\right.
$$

where $f(t, u, v)$ is a Carathéodory function.

A function $f:[0,2 \pi] \times \mathbb{R}^{2} \rightarrow \mathbb{R}$ is said to be a Carathéodory function if $f(t, \cdot, \cdot)$ is continuous for a.e. $t \in[0,2 \pi], f(\cdot, u, v)$ is measurable for any $(u, v) \in \mathbb{R}^{2}$ and for each constant $r>0$ there exists a function $h_{r}(t) \in$ $L^{1}[0,2 \pi]$ such that $|f(t, u, v)| \leq h_{r}(t)$ for a.e. $t \in[0,2 \pi]$ with $\max \{|u|,|v|\}$ $\leq r$.

We say that $\alpha, \beta \in W^{2,1}[0,2 \pi]$ are lower and upper solutions to (1.1), respectively, if they satisfy

$$
\left\{\begin{array}{l}
\alpha^{\prime \prime}(t) \geq f\left(t, \alpha(t), \alpha^{\prime}(t)\right) \quad \text { for a.e. } t \in[0,2 \pi] \\
\alpha(0)=\alpha(2 \pi), \quad \alpha^{\prime}(0) \geq \alpha^{\prime}(2 \pi)
\end{array}\right.
$$

and

$$
\left\{\begin{array}{l}
\beta^{\prime \prime}(t) \leq f\left(t, \beta(t), \beta^{\prime}(t)\right) \quad \text { for a.e. } t \in[0,2 \pi] \\
\beta(0)=\beta(2 \pi), \quad \beta^{\prime}(0) \leq \beta^{\prime}(2 \pi) .
\end{array}\right.
$$

We call a function $u \in W^{2,1}[0,2 \pi]$ a solution of the problem (1.1) if it is both a lower and an upper solution of (1.1).

2000 Mathematics Subject Classification: 34B10, 34B15.

Key words and phrases: nonlinear periodic boundary value problem, monotone method, extremal solutions, lower and upper solutions. 
Under the classical assumption that $\alpha(t) \leq \beta(t)$, a number of authors have studied the existence of solutions of second order periodic boundary value problem by means of the method of lower and upper solutions or the monotone iterative technique (see [1, 3-7, 9-12, 17, 19]). Only a few have dealt with the case where $\alpha(t), \beta(t)$ satisfy the opposite ordering condition $\beta(t) \leq \alpha(t)$ (see $[2,8,13-16,19]$ ), and if $\beta(t) \leq \alpha(t)$ the monotone method is not valid in general (see $[2,8,13,14,16])$. Recently, Wang [18] has investigated a special case of $(1.1)$ (where $f(t, u, v)=-k v-g(t, u)$ and $g(t, u)$ is nonincreasing with respect to $u$ ) in the presence of a lower solution $\alpha(t)$ and an upper solution $\beta(t)$ with $\beta(t) \leq \alpha(t)$. Moreover, Rachůnková [15] has recently proved that the problem (1.1) has at least one solution $u(t)$ in the case $\beta(t) \leq \alpha(t)$. However, the proof in [15] was not constructive, it did not guarantee that $u(t)$ satisfies $\beta(t) \leq u(t) \leq \alpha(t)$.

The purpose of this paper is to prove the existence of solutions of (1.1) under the assumption that there exist a lower solution $\alpha(t)$ and an upper solution $\beta(t)$ of $(1.1)$ with $\beta(t) \leq \alpha(t)$. We will develop the monotone iterative method to approximate the extremal solution of (1.1) and prove that the solution $u(t)$ of $(1.1)$ satisfies $\beta(t) \leq u(t) \leq \alpha(t)$. Our result extends and complements those in $[15,18]$.

The following hypotheses are adopted throughout this paper:

$\left(\mathrm{H}_{1}\right)$ For given $\alpha, \beta \in C[0,2 \pi]$ with $\beta(t) \leq \alpha(t)$ on $[0,2 \pi]$ there exists $0<L<|M|$ such that

$$
(M-L)\left(v_{2}-v_{1}\right) \leq f\left(t, u, v_{1}\right)-f\left(t, u, v_{2}\right) \leq(M+L)\left(v_{2}-v_{1}\right)
$$

for a.e. $t \in[0,2 \pi]$ whenever $\beta(t) \leq u \leq \alpha(t), v_{2} \geq v_{1}, v_{1}, v_{2} \in \mathbb{R}$.

$\left(\mathrm{H}_{2}\right)$ The inequality $f\left(t, u_{2}, v\right)-f\left(t, u_{1}, v\right) \geq 0$ holds for a.e. $t \in[0,2 \pi]$ whenever $\beta(t) \leq u_{1} \leq u_{2} \leq \alpha(t), v \in \mathbb{R}$.

The main result of this paper is as follows.

THEOREM 1. Assume that there exists a lower solution $\alpha(t)$ and an upper solution $\beta(t)$ of $(1.1)$ such that $\beta(t) \leq \alpha(t)$ on $[0,2 \pi]$, and $f(t, u, v)$ is a Carathéodory function satisfying hypotheses $\left(\mathrm{H}_{1}\right),\left(\mathrm{H}_{2}\right)$. Then there exist two sequences $\left\{\beta_{j}\right\}$ and $\left\{\alpha_{j}\right\}$, nondecreasing and nonincreasing, respectively, with $\beta_{0}=\beta$ and $\alpha_{0}=\alpha$, which converge uniformly and monotonically to the extremal solution to the problem (1.1) in the interval $[\beta, \alpha]:=\{u \in C[0,2 \pi]$ : $\beta(t) \leq u(t) \leq \alpha(t)$ on $[0,2 \pi]\}$.

2. Maximum-minimum principle. To prove the validity of the monotone iterative technique, we present the following maximum-minimum principle. 
Lemma 2. Let $y \in W^{1,1}[0,2 \pi]$ satisfy

$$
\left\{\begin{array}{l}
y^{\prime}(t)+M y(t)+L|y(t)| \geq 0 \quad \text { for a.e. } t \in[0,2 \pi], \\
y(0) \geq y(2 \pi)
\end{array}\right.
$$

where $|M|>L \geq 0$. Then $M y(t) \geq 0$ on $[0,2 \pi]$, i.e. $\min _{t \in[0,2 \pi]} y(t) \geq 0$ when $M>0$ and $\max _{t \in[0,2 \pi]} y(t) \leq 0$ when $M<0$.

Proof. Let $M>0$. Suppose to the contrary that $y(t)<0$ for some $t \in[0,2 \pi]$. It is enough to consider the following three cases.

CASE (i): $y(t)<0$ on $[0,2 \pi]$. In this case, we have $y^{\prime}(t) \geq(L-M) y(t)$ $>0$ for a.e. $t \in[0,2 \pi]$, and hence $y(0)<y(2 \pi)$, which contradicts the fact that $y(0) \geq y(2 \pi)$.

CASE (ii): $y(2 \pi) \geq 0$ and $y(t)<0$ for some $t \in[0,2 \pi]$. Since $y(0) \geq$ $y(2 \pi) \geq 0$, there exists an interval $(a, b), 0 \leq a<b \leq 2 \pi$, such that $y(t)<0$ in $(a, b)$ and $y(a)=y(b)=0$. Therefore, we have $y^{\prime}(t) \geq(L-M) y(t)>0$ for a.e. $t \in(a, b)$, and hence $y(a)<y(b)$, which is a contradiction.

CASE (iii): $y(2 \pi)<0$ and $y(t) \geq 0$ for some $t<2 \pi$. In this case, there exists a point $t_{0} \in[0,2 \pi]$ such that $y(t)<0$ in $\left(t_{0}, 2 \pi\right]$, and $y\left(t_{0}\right)=0$. As a result, we have $y^{\prime}(t) \geq(L-M) y(t)>0$ for a.e. $t \in\left(t_{0}, 2 \pi\right]$, and hence $y\left(t_{0}\right)<y(2 \pi)<0$, which is also a contradiction. This shows that $y(t) \geq 0$ on $[0,2 \pi]$ when $M>0$.

In very much the same way, we can prove that $y(t) \leq 0$ on $[0,2 \pi]$ when $M<0$. The proof of the lemma is complete.

Lemma 2 is an improvement and extension of Lemma 1.2.2 of [7].

3. Proof of Theorem 1. We first consider the case $M>0$. For each given $\eta \in[\beta, \alpha]$, we study the following periodic boundary value problem:

$$
\left\{\begin{array}{l}
u^{\prime \prime}(t)+M u^{\prime}(t)=g\left(t, \eta(t), u^{\prime}(t)\right), \quad t \in[0,2 \pi], \\
u(0)=\eta(2 \pi), \quad u^{\prime}(0)=u^{\prime}(2 \pi),
\end{array}\right.
$$

where

$$
g\left(t, \eta(t), u^{\prime}(t)\right):=f\left(t, \eta(t), u^{\prime}(t)\right)+M u^{\prime}(t) .
$$

Let $u$ be a solution of $(3.1)$ and $v(t)=u^{\prime}(t)$. We get

$$
\left\{\begin{array}{l}
v^{\prime}(t)+M v(t)=g(t, \eta(t), v(t)), \quad t \in[0,2 \pi] \\
v(0)=v(2 \pi)
\end{array}\right.
$$

It is easy to see that the above problem is equivalent to the integral equation

$$
v(t)=\int_{0}^{2 \pi} G(t, s) g(s, \eta(s), v(s)) d s,
$$


where

$$
G(t, s)= \begin{cases}\frac{e^{M(2 \pi+s-t)}}{e^{2 M \pi}-1}, & 0 \leq s \leq t \leq 2 \pi, \\ \frac{e^{M(s-t)}}{e^{2 M \pi}-1}, & 0 \leq t \leq s \leq 2 \pi .\end{cases}
$$

Let $v$ be a solution of the problem (3.4). Then

$$
u(t):=\eta(2 \pi)+\int_{0}^{t} v(s) d s
$$

is certainly a solution to the problem (3.1).

Lemma 3. For each fixed $\eta \in[\beta, \alpha]$, the problem (3.1) has a unique solution $u \in W^{2,1}[0,2 \pi]$.

Proof. Define the mapping $T: C[0,2 \pi] \rightarrow C[0,2 \pi]$ by

$$
(T v)(t):=\int_{0}^{2 \pi} G(t, s) g(s, \eta(s), v(s)) d s .
$$

By $\left(\mathrm{H}_{1}\right)$ we have

$$
\left|g\left(t, \eta, v_{2}\right)-g\left(t, \eta, v_{1}\right)\right| \leq L\left|v_{2}-v_{1}\right|
$$

for any $v_{1}, v_{2} \in C[0,2 \pi]$ whenever $\beta(t) \leq \eta \leq \alpha(t)$, and hence

$$
\begin{aligned}
\left|\left(T v_{2}\right)(t)-\left(T v_{1}\right)(t)\right| & \leq \int_{0}^{2 \pi}|G(t, s)| \cdot\left|g\left(s, \eta(s), v_{2}(s)\right)-g\left(s, \eta(s), v_{1}(s)\right)\right| d s \\
& \leq L\left\|v_{2}-v_{1}\right\| \int_{0}^{2 \pi}|G(t, s)| d s=\frac{L}{M}\left\|v_{2}-v_{1}\right\|,
\end{aligned}
$$

i.e.

$$
\left\|T v_{2}-T v_{1}\right\| \leq \frac{L}{M}\left\|v_{2}-v_{1}\right\|
$$

where $\|u\|=\max \{|u(t)|: 0 \leq t \leq 2 \pi\}$; this shows that $T$ is a contraction mapping. The Banach contraction principle tells us that $T$ has a unique fixed point $v$ in $C[0,2 \pi]$ and $v$ satisfies the integral equation (3.4). Therefore, the function $u$ defined by (3.5) is a unique solution of (3.1). The proof is complete.

We define a mapping $\Phi:[\beta, \alpha] \rightarrow W^{2,1}[0,2 \pi]$ by setting $(\Phi \eta)(t):=u(t)$, where $u(t)$ is the unique solution of the problem (3.1) with given $\eta \in[\beta, \alpha]$. Then it follows by Lemma 3 that the mapping $\Phi$ is well defined.

Concerning the mapping $\Phi$, the following statement holds:

LEMma 4. The mapping $\Phi$ has the following properties:

(i) $\beta(t) \leq(\Phi \beta)(t),(\Phi \alpha)(t) \leq \alpha(t)$ on $[0,2 \pi]$; 
(ii) $\Phi$ is increasing on $[\beta, \alpha]$, namely, $\left(\Phi \eta_{1}\right)(t) \leq\left(\Phi \eta_{2}\right)(t)$ on $[0,2 \pi]$ when $\eta_{1}, \eta_{2} \in[\beta, \alpha]$ and $\eta_{1}(t) \leq \eta_{2}(t)$ on $[0,2 \pi]$.

Proof. (i) Set $\beta_{1}(t):=(\Phi \beta)(t), x(t):=\beta_{1}(t)-\beta(t)$, and $y(t):=x^{\prime}(t)$. It follows from $\left(\mathrm{H}_{1}\right)$ and (1.3) that

$$
\left\{\begin{aligned}
x^{\prime \prime}(t)+M x^{\prime}(t) & \geq g\left(t, \beta(t), \beta_{1}^{\prime}(t)\right)-g\left(t, \beta(t), \beta^{\prime}(t)\right) \\
& \geq-L\left|x^{\prime}(t)\right| \text { for a.e. } t \in[0,2 \pi] \\
x(0)=0, \quad x^{\prime}(0) & \geq x^{\prime}(2 \pi)
\end{aligned}\right.
$$

i.e.

$$
\left\{\begin{array}{l}
y^{\prime}(t)+M y(t)+L|y(t)| \geq 0 \quad \text { for a.e. } t \in[0,2 \pi], \\
y(0) \geq y(2 \pi) .
\end{array}\right.
$$

Applying Lemma 2 we conclude that $M y(t) \geq 0$ on $[0,2 \pi]$. Thus we have $x(t)=\int_{0}^{t} y(s) d s \geq 0$ for $t \in[0,2 \pi]$. That is, $\beta(t) \leq(\Phi \beta)(t)$ on $[0,2 \pi]$. A similar argument shows that $(\Phi \alpha)(t) \leq \alpha(t)$ on $[0,2 \pi]$. This proves (i).

(ii) Let $u_{j}(t):=\left(\Phi \eta_{j}\right)(t), j=1,2, x(t):=u_{2}(t)-u_{1}(t), y(t):=x^{\prime}(t)$, where $\eta_{1}, \eta_{2} \in[\beta, \alpha]$ and $\eta_{1}(t) \leq \eta_{2}(t)$ on $[0,2 \pi]$. By $\left(\mathrm{H}_{1}\right),\left(\mathrm{H}_{2}\right)$ we have

$$
\left\{\begin{aligned}
x^{\prime \prime}(t)+M x^{\prime}(t) & =g\left(t, \eta_{2}(t), u_{2}^{\prime}(t)\right)-g\left(t, \eta_{1}(t), u_{1}^{\prime}(t)\right) \\
& \geq-L\left|x^{\prime}(t)\right| \quad \text { for a.e. } t \in[0,2 \pi], \\
x(0) \geq 0, \quad x^{\prime}(0) & =x^{\prime}(2 \pi),
\end{aligned}\right.
$$

i.e.

$$
\left\{\begin{array}{l}
y^{\prime}(t)+M y(t)+L|y(t)| \geq 0 \quad \text { for a.e. } t \in[0,2 \pi] \\
y(0)=y(2 \pi) .
\end{array}\right.
$$

Applying Lemma 2 as before, we get $y(t) \geq 0$ on $[0,2 \pi]$. Thus we have $x(t)=x(0)+\int_{0}^{t} y(s) d s \geq 0$ for $t \in[0,2 \pi]$. This shows that $\left(\Phi \eta_{2}\right)(t) \geq$ $\left(\Phi \eta_{1}\right)(t)$ when $\eta_{1}, \eta_{2} \in[\beta, \alpha]$ and $\eta_{2}(t) \geq \eta_{1}(t)$ on $[0,2 \pi]$, which proves (ii).

We now consider the case $M<0$. For each given $\eta \in[\beta, \alpha]$, we study the following second order periodic boundary value problem:

$$
\left\{\begin{array}{l}
u^{\prime \prime}(t)+M u^{\prime}(t)=g\left(t, \eta(t), u^{\prime}(t)\right), \\
u(2 \pi)=\eta(0), \quad u^{\prime}(0)=u^{\prime}(2 \pi),
\end{array}\right.
$$

where $\eta \in[\beta, \alpha]$ and $g(t, u, v)$ is given by (3.2). As in Lemma 3 it can be proved that

$$
u(t)=\eta(0)-\int_{t}^{2 \pi} v(s) d s
$$

is a solution of the problem (3.6), where $v(s)$ is a solution of (3.4) for $M<0$. Let $y(t):=x^{\prime}(t), x(t):=\beta_{1}(t)-\beta(t), \beta_{1}(t):=(\Phi \beta)(t)$. Then as in Lemma 4 we can easily prove $y(t) \leq 0$ on $[0,2 \pi]$ and so $x(t)=x(2 \pi)-\int_{t}^{2 \pi} y(s) d s \geq 0$. Thus, all results of Lemma 4 still hold when $M<0$. 
Let us define sequences $\left\{\beta_{j}\right\}$ and $\left\{\alpha_{j}\right\}$ such that

$$
\beta_{j+1}(t):=\left(\Phi \beta_{j}\right)(t), \quad \alpha_{j+1}(t):=\left(\Phi \alpha_{j}\right)(t)
$$

with $\beta_{0}=\beta, \alpha_{0}=\alpha$. From Lemma 4 , we conclude that $\left\{\beta_{j}\right\}$ is nondecreasing and $\left\{\alpha_{j}\right\}$ nonincreasing. It is clear that $\left\{\alpha_{j}\right\}_{j=1}^{\infty}$ and $\left\{\beta_{j}\right\}_{j=1}^{\infty}$ are bounded, and hence there exists an $r>0$ such that $\left|\alpha_{j}\right| \leq r$ and $\left|\beta_{j}\right| \leq r$ for all $j$. Since

$$
\begin{aligned}
\alpha_{j+1}^{\prime}(t) & =\int_{0}^{2 \pi} G(t, s) g\left(s, \alpha_{j}(s), \alpha_{j+1}^{\prime}(s)\right) d s \\
& =\int_{0}^{2 \pi} G(t, s)\left[g\left(s, \alpha_{j}(s), \alpha_{j+1}^{\prime}(s)\right)-g\left(s, \alpha_{j}(s), 0\right)+g\left(s, \alpha_{j}(s), 0\right)\right] d s,
\end{aligned}
$$

by $\left(\mathrm{H}_{1}\right)$ we have

$$
\begin{aligned}
\left\|\alpha_{j+1}^{\prime}\right\| \leq & \max _{t \in[0,2 \pi]} \int_{0}^{2 \pi}|G(t, s)|\left[\left|g\left(s, \alpha_{j}(s), \alpha_{j+1}^{\prime}(s)\right)-g\left(s, \alpha_{j}(s), 0\right)\right|\right. \\
& \left.+\left|f\left(s, \alpha_{j}(s), 0\right)\right|\right] d s \\
\leq & L\left\|\alpha_{j+1}^{\prime}\right\| \max _{t \in[0,2 \pi]} \int_{0}^{2 \pi}|G(t, s)| d s+\max _{t \in[0,2 \pi]} \int_{0}^{2 \pi}|G(t, s)| h_{r}(s) d s \\
\leq & \frac{L}{|M|}\left\|\alpha_{j+1}^{\prime}\right\|+\frac{\max \left\{e^{2 M \pi}, 1\right\}}{\left|e^{2 M \pi}-1\right|} \int_{0}^{2 \pi} h_{r}(s) d s,
\end{aligned}
$$

and hence

$$
\left\|\alpha_{j+1}^{\prime}\right\| \leq \frac{|M| \max \left\{e^{2 M \pi}, 1\right\}}{(|M|-L)\left|e^{2 M \pi}-1\right|} \int_{0}^{2 \pi} h_{r}(s) d s .
$$

This shows that $\left\{\alpha_{j}^{\prime}\right\}_{j=1}^{\infty}$ is bounded. Analogously, $\left\{\beta_{j}^{\prime}\right\}_{j=1}^{\infty}$ is also bounded. It then follows by a standard argument (see e.g. [7]) that

$$
\lim _{j \rightarrow \infty} \beta_{j}(t)=: \beta^{*}(t), \quad \lim _{j \rightarrow \infty} \alpha_{j}(t)=: \alpha^{*}(t)
$$

uniformly and monotonically on $[0,2 \pi]$. From the integral representations (3.4), (3.5) and (3.7), we conclude that $\beta^{*}(t)$ and $\alpha^{*}(t)$ are both solutions of the problem (3.1) or (3.6), and hence they are solutions of (1.1).

Furthermore, if $u \in[\beta, \alpha]$ is a solution to the problem (1.1) then, by induction, $\beta_{j}(t) \leq u(t) \leq \alpha_{j}(t)$ on $[0,2 \pi], j=0,1,2, \ldots$, and hence $u \in$ $\left[\beta^{*}, \alpha^{*}\right]$. This shows that $\beta^{*}(t)$ and $\alpha^{*}(t)$ are respectively minimal and maximal solutions of the problem (1.1) in the segment $[\beta, \alpha]$.

This completes the proof of Theorem 1 . tion:

REMARK. The hypothesis $\left(\mathrm{H}_{1}\right)$ can be replaced by the following condi- 
$\left(\mathrm{H}_{1}^{*}\right)$ For given $\beta, \alpha \in C[0,2 \pi]$ with $\beta(t) \leq \alpha(t)$ on $[0,2 \pi]$, there exist $0<A<B$ such that

$$
\begin{aligned}
A\left(v_{2}-v_{1}\right) & \leq f\left(t, u, v_{1}\right)-f\left(t, u, v_{2}\right) \leq B\left(v_{2}-v_{1}\right) \quad \text { or } \\
-B\left(v_{2}-v_{1}\right) & \leq f\left(t, u, v_{1}\right)-f\left(t, u, v_{2}\right) \leq-A\left(v_{2}-v_{1}\right)
\end{aligned}
$$

whenever $\beta(t) \leq u \leq \alpha(t), v_{2} \geq v_{1}, v_{1}, v_{2} \in \mathbb{R}$.

\section{References}

[1] A. Cabada, The method of lower and upper solutions for second, third, fourth, and higher order boundary value problems, J. Math. Anal. Appl. 185 (1994), 302-320.

[2] - The monotone method for boundary value problems, doctoral thesis, Universidad Santiago de Compostela, 1992 (in Spanish).

[3] A. Cabada and J. J. Nieto, A generalization of the monotone iterative technique for nonlinear second order periodic boundary value problems, J. Math. Anal. Appl. 151 (1990), 181-189.

[4] - - - Extremal solutions of second order nonlinear periodic boundary value problems, Appl. Math. Comput. 40 (1990), 135-145.

[5] W. J. Gao and J. Y. Wang, On a nonlinear second order periodic boundary value problem with Carathéodory functions, Ann. Polon. Math. 62 (1995), 283-291.

[6] D. Q. Jiang and J. Y. Wang, A generalized periodic boundary value problem for the one-dimensional p-Laplacian, ibid. 65 (1997), 265-270.

[7] G. S. Ladde, V. Lakshmikantham and A. S. Vatsala, Monotone Iterative Techniques for Nonlinear Differential Equations, Pitman, Boston, 1985.

[8] J. J. Nieto, Nonlinear second order periodic boundary value problems, J. Math. Anal. Appl. 130 (1988), 22-29.

[9] - Nonlinear second order periodic boundary value problems with Carathéodory functions, Appl. Anal. 34 (1989), 111-128.

[10] —, Periodic solutions for third order ordinary differential equations, Comment. Math. Univ. Carolin. 32 (1991), 495-499.

[11] J. J. Nieto and A. Cabada, A generalized upper and lower solution method for nonlinear second order ordinary differential equations, J. Appl. Math. Stochastic Anal. 5 (1992), 157-166.

[12] P. Omari, A monotone method for constructing extremal solutions for second order scalar boundary value problems, Appl. Math. Comput. 18 (1986), 257-275.

[13] —, Nonordered lower and upper solutions and solvability of the periodic problem for the Liénard and the Rayleigh equations, Rend. Istit. Mat. Univ. Trieste 20 (1991), $54-64$.

[14] P. Omari and M. Trombetta, Remarks on the lower and upper solutions method for second and third-order periodic boundary value problems, Appl. Math. Comput. 50 (1992), 1-21.

[15] I. Rachůnková, Upper and lower solutions satisfying the inverse inequality, Ann. Polon. Math. 65 (1997), 235-244.

[16] B. Rudolf and Z. Kubáček, Remarks on J. J. Nieto's paper: "Nonlinear second order periodic boundary value problems", J. Math. Anal. Appl. 146 (1990), 203-206.

[17] V. Šeda, J. J. Nieto and M. Gera, Periodic boundary value problems for nonlinear higher order ordinary differential equations, Appl. Math. Comput. 48 (1992), 71-82. 
[18] C. G. Wang, Generalized upper and lower solution method for the forced Duffing equation, Proc. Amer. Math. Soc. 125 (1997), 397-406.

[19] M. X. Wang, A. Cabada and J. J. Nieto, Monotone method for nonlinear second order periodic boundary value problems with Carathéodory functions, Ann. Polon. Math. 58 (1993), 221-235.

Department of Mathematics

Northeast Normal University

Changchun 130024, P.R. China
Department of Mathematics

Daqing Petroleum Institute

Anda 151400, Heilongjiang, P.R. China

Reçu par la Rédaction le 14.7.2000 